\title{
Optimal Inventory Strategies for Pharmaceutical Products Incorporating Carbon Emissions
}

\author{
Priyan $\mathrm{S}^{*}$ \\ Department of Mathematics, Mepco Schlenk Engineering College, India \\ Submission: April 25, 2019; Published: May 28, 2019 \\ *Corresponding author: Priyan S, Department of Mathematics, Mepco Schlenk Engineering College, Virudhunagar 626005, Tamilnadu, India
}

\begin{abstract}
Health sector is a significant source of carbon emissions around the world. Today healthcare industries are looking for solutions to reduce carbon emissions associated with their operations. Operational adjustments, such as modifications in inventory strategies, have proven to be an effective way to decrease emissions. This paper offers optimal inventory strategies for healthcare system to achieve target customer service level while minimizing system total cost and carbon emissions.
\end{abstract}

Keywords: Pharmaceutical; Optimal decisions; Carbon emission

Abbreviations: HSC: Healthcare Supply Chain; SLC: Service Level Constraint

\section{Introduction}

A healthcare supply chain (HSC) consists of pharmaceutical wholesalers, pharmaceutical companies, hospitals, medical surgical distributors, etc. A stated one-third of hospital decision makers have belief their hospital supply chain is working at greatest point doing work well, but the other two thirds are discovering themselves one foot in fee-for-service and the other in value-based giving back of money. Many of these hospitals are not implementing the suitable supply chain management strategies. This is because most hospital administrators and pharmacy managers are doctors with expert knowledge in medicine and are not supply chain professionals. Unlike other industries, hospital administrators and pharmacy managers have to manage very complicated distribution networks and inventory management problems without proper guidance on efficient practices. Hence, given the high costs, constraints, and perishability of pharmaceuticals, more study is necessary to help health care managers in setting optimal HSC strategies. Recently few numbers of papers designed in the field of healthcare supply chain and inventory management (see [1-4]).

All steps from the supply of raw materials to the finished products can be covered in a supply chain connecting raw material suppliers, manufacturers, retailers, and the customer/ hospital. Multi-echelon inventory management is the management of inventory and coordination of the distribution process at more than one level of a supply chain network. Kim [5] presented an explanation of an integrated supply chain management system developed to specifically address issues related to pharmaceuticals in the health care sector. Meijboom and Obel [6] addressed supply chain coordination for a pharmaceutical company with a multi-location and multi-stage operations structure. Amir et al. [7] developed a generalized network oligopoly model for HSC competition that takes into account product perishability, brand differentiation, and discard costs.

Lead time is an essential factor in any supply chain and inventory system. Liao \& Shyu [8] devised a probabilistic inventory model in which lead time was considered as a decision variable. Several researchers extended this approach in integrated inventory models for lead time reduction (see [914]). Glock [13] studied alternative methods for controlling lead time and their impact on the safety stock and the expected total costs of a continuous review inventory control system. Moua et al. [14] introduced a more realistic lead time crashing cost in Glock [13]'s model. They proposed a modified integrated inventory model by adding the transportation time as a decision variable and assumed that there were two different safety stocks. They numerically showed that their modified model was more realistic than Glock [13] model.

Without considering carbon emissions in inventory management, the decision objective is usually set as the total cost minimization or the total profit maximization. However, taking emission reduction into account is likely to change the optimal solution and incurs additional cost. The health sector is a vital direct source of carbon emissions. It is also a highly 
heterogeneous segment that has seen both rapid increases and decreases in direct emissions from different sources over the last decade. A modification in inventory strategies is an effective way to decrease emissions so that carbon emission issues in inventory management have attracted attention in literature recently (see [15-19]).

This research explores the impact of both lead time and carbon emissions on the inventory decisions of a pharmaceutical company and hospital supply chain system. The paper assumes that the lead time for the first shipment consists of production time, setup and transportation time (nonproductive time), while the lead time for the rest of the shipments is only transportation time, and that lead time can be reduced by shortening nonproductive time and transportation time. The proposed study also adds the carbon emissions from transportation and storage into the account. The intention of our investigation is to obtain the optimal decisions (order quantity, production rate, nonproductive time, transportation time and total number of deliveries) to achieve hospital's CSL while minimizing expected total cost, and to examine the effects of carbon emissions in the proposed healthcare system.

\section{Notations and Assumptions}

To develop the proposed model, we adopt the following notations and assumptions which are similar to those used in Uthayakumar \& Priyan [1]

\section{Notations}

I. For the Hospital

$D \quad$ Expected demand rate in units per unit time

$\sigma \quad$ The standard deviation of the demand in units per unit time

A Ordering cost per order.

$h \quad$ Holding cost per unit time

$S_{p} \quad$ Procurement cost per unit

$Q \quad$ Order quantity (finished products), a decision variable

$\omega$ The expiration time (or shelf life), which is the length of time to the expiration date.

$\theta(t)$ Deteriorating rate of finished products at time t, $0 \leq$ $\theta(t) \leq 1$

\section{$L \quad$ Length of lead time, a decision variable}

$n \quad$ The number of lots in which the product is delivered from the company to the hospital in one production cycle, a positive integer, a decision variable

$\alpha \quad$ Proportion of demands that are not met from stock so $(1-\alpha)$ is the service level

$X \quad$ The lead time demand in units per unit time, a random variable
II. For the pharmaceutical company

$P \quad$ Production rate in units per unit time

$S \quad$ Company's setup cost per setup

$h_{p} \quad$ Holding cost per unit time.

\section{Model Development}

\section{Hospital's expected total cost}

The hospital places an order after every $Q$ units, therefore for expected ordering cycle time of $Q / D$, the expected ordering cost per unit time can be expressed by $\frac{A D}{Q}$. Similar to Glock [13], we assume that the nonproductive time $t$ consists of $m$ mutually independent components $t_{s r}$, i.e. $t_{s}=\sum_{r=1}^{m^{s}} t_{s r}$. The $r^{t h}$ component has a normal duration $u_{r}$ a minimum duration $v_{r}$ and a crashing cost per unit time $c_{r^{\prime}}$. For convenience, we rearrange $c_{r}$ such that $c_{1} \leq c_{2} \leq \ldots \leq c_{m}$. Let $t_{s}^{i}$ represent the length of nonproductive time with the components $1,2, \ldots i$ crashed to their minimum duration, then $t_{s}^{i}$ can be expressed as $t_{s}^{i}=\sum_{r=1}^{m} u_{r}-\sum_{r=1}^{i}\left(u_{r}-v_{r}\right)$, $i=1,2, \ldots . m$; and the lead time crashing cost per cycle is $C\left(t_{s}\right)=c_{i}\left(t_{s}^{i-1}-t_{s}\right)+\sum_{r=1}^{i-1} c_{r}\left(u_{r}-v_{r}\right), t_{s} \in\left[t_{s}^{i}, t_{s}^{i-1}\right]$

To gain the transportation time crashing cost $C\left(t_{T}\right)$ for the shipments $1,2,3, \ldots . n$. Similar to the assumptions made by Mou [14], we assume that the $\mathrm{l}^{\text {th }}$ component of the nonproductive time $t_{s}$ is the setup time. Thus, the transportation time $t_{T}$ is composed of $m-1$ mutually independent components, i.e.

$t_{T}=\sum_{r=1}^{m} t_{s r}$. We rearrange $c_{1}, c_{2}, \ldots, c_{i-1}, c_{l+1}, \ldots . c_{m}$ in such a way that $c_{1}^{\prime} \leq c_{2}^{\prime} \leq \cdots \leq c_{m-1}^{\prime}$. Let $t_{T}^{j}$ be the length of the transportation time with the components $1,2,3, \ldots j$ crashed to their minimum duration, then $t_{T}^{j}$ can be expressed as $t_{T}^{j}=\sum_{r=1}^{m-1} u_{r}^{\prime}-\sum_{r=1}^{j}\left(u_{r}^{\prime}-v_{r}^{\prime}\right), \quad 1,2,3, . . m-1$. Hence, by adopting the same technique used by Mou [14], the transportation time crashing cost $C\left(t_{T}\right)$ for the shipments $2,3, \ldots n$ $C\left(t_{T}\right)=c_{i}^{\prime}\left(t_{T}^{j-1}-t_{T}\right)+\sum_{r=1}^{j-1} c_{r}^{\prime}\left(u_{r}^{\prime}-v_{r}^{\prime}\right), t_{T} \in\left[t_{T}^{j}, t_{T}^{j-1}\right]$.

Thus the lead time crashing cost per unit time can be derived by $\frac{D}{n Q}\left[R\left(t_{s}\right)+(n-1) R\left(t_{T}\right)\right]$. In addition, the reduction of lead time leads to a lower demand uncertainty, which may decrease the safety stock and the stock-out loss. Since $\frac{Q}{P}+t_{s} \gg t_{T}$, for the shipments $2,3, \ldots n$ it might be beneficial to avoid holding additional safety stock by decreasing the reorder points. Consequently, Similar to Mou [14], we consider that there are two different safety stocks and reorder points for the first shipment and the shipments $2,3, \ldots n$. Then, the hospital's expected holding cost per unit time is

$$
H_{b}\left(Q, P, n, t_{s}, t_{T}\right)=h_{b}\left(\frac{Q}{2}+\frac{1}{n} k_{1} \delta \sqrt{\frac{Q}{P}+t_{s}}+\frac{n-1}{n} k_{2} \delta \sqrt{t_{T}}\right)
$$

The hospital's freshness index of a pharmaceutical product (perishable product) may be pretentious by time, temperature, dampness, refrigeration, etc. In practice, most pharmaceutical products have their own expiration dates. Feng et al. stated that health-conscious consumers prefer a perishable product with a longer remaining shelf life to a shorter one and they assumed that the customer's freshness index is age-dependent. That is, it 
begins with 1 at time 0 , and then gradually degrades closer to 0 as the expiration date of the product approaches. Consequently, similar to Feng et al., we assume that the age-dependent freshness at time tis linearly decreasing from 1 at the beginning to 0 at the maximum lifetime $m$ as follows:

$$
\theta(t)=\frac{\omega-t}{\omega}, 0 \leq t \leq \omega .
$$

Subsequently the expired finished products of the hospital for the ordering cycle $\frac{Q}{D}$ can be detected by $Q \int_{0}^{\frac{Q}{D}} \theta(t) d t=\frac{Q^{2}}{D}\left(1-\frac{Q}{2 D \omega}\right)$ and disposed it with dispose cost $\frac{\varepsilon}{Q^{2}}$ since the hospital's safety stock inventories are sufficient to replace these expired finished products. Then similar to the existing models, we consider the reorder point $r=D L+k \sigma \sqrt{L}$ where kis the safety factor. Hence, hospital's expected total cost per unit time is composed of ordering cost, holding cost, disposal cost and crashing cost, is expressed by

$$
\begin{gathered}
\operatorname{HETC}_{b}\left(Q, P, n, t_{s}, t_{T}\right) \\
=\frac{A D}{Q}+h_{b}\left(\frac{Q}{2}+\frac{1}{n} k_{1} \delta \sqrt{\frac{Q}{P}+t_{s}}+\frac{n-1}{n} k_{2} \delta \sqrt{t_{T}}\right)+\frac{\varepsilon}{D}\left(1-\frac{Q}{2 D \omega}\right) \\
+\frac{D}{n Q}\left[R\left(t_{s}\right)+(n-1) R\left(t_{T}\right)\right]
\end{gathered}
$$

We assume in view of realistic healthcare system that the hospital has set a target service level in terms of the fill rate corresponding to the proportion of all product demands to be satisfied directly from available stock. Therefore, the Service Level Constraint (SLC) imposes a limit on the proportion of demands that are not met from stock. This can be mathematically expressed as

$$
\frac{E(X-r)^{+}}{Q} \leq \alpha
$$

We consider the demand during lead time is normally distributed with mean $D L(P, Q)$ and $D L\left(t_{T}\right.$ ), respectively, and standard deviation $\sigma \sqrt{L(p, Q)}$ and $\sigma{\sqrt{L\left(t_{T}\right)}}^{T}$, respectively, the safety stock is given by $S_{s}=k_{1} \sigma \sqrt{t_{s}+\frac{Q}{P}}$. The expected shortage of the first batch shipment is given as $\int_{s_{1}}^{\infty}\left(x_{1}-s_{1}\right) f\left(x_{1}\right) d x_{1}=\sigma \sqrt{t_{s}+\frac{Q}{P}} \psi\left(k_{1}\right)$. For batches $2,3, \ldots n$, the expected shortage amount is $\int_{s_{2}}^{\infty}\left(x_{2}-s_{2}\right) f\left(x_{1}\right) d x_{2}=\sigma \sqrt{t_{T}} \psi\left(k_{2}\right) . \psi\left(k_{i}\right)$ is the loss function, which can be calculated as $\psi\left(k_{i}\right)=\int_{k_{i}}^{\infty}\left(z-k_{i}\right) \varphi(z) d z$ where $\varphi(z)$ is the standard normal probability density function. Then, the safety stock $S_{s}$ can also be expressed as $S_{s}=k_{2} \sigma \sqrt{t_{T}}$.

Now SLC can be written $\sigma\left(\sqrt{t_{s}+\frac{Q}{P}} \psi\left(k_{1}\right)+\sqrt{t_{T}} \psi\left(k_{2}\right)\right) \leq \alpha Q$

\section{Pharmaceutical company's expected total cost}

Once the hospital orders a lot size of $Q$ units, the company produces the products in a lot size of $n Q$ units with the setup cost $S$ in each production cycle of length $\frac{n Q}{D}$ with constant production rate $P$ units per unit time, and the hospital will receive the supply in n lots each of size $Q$ units. The first lot size of $Q$ units is ready for shipment after time $\frac{Q}{P}$ just after the start of the production. During the production run time, $\frac{n Q}{P}$, the company's inventory is building up at a constant rate, and simultaneously supplies a lot of size $Q$ units to the buyer on expected every $\frac{Q}{D}$ units of time.
Subsequently, during the non-production period the company continues their shipments to the hospital on expected every $\frac{Q}{D}$ units of time (ordering cycle) until the inventory level falls to zero. Based on the existing inventory models, the company's expected on hand finished products inventory is evaluated as the difference of the company's accumulated inventory and the hospital's accumulated inventory. Now, according to Uthayakumar \& Priyan [1], company's accumulated inventory is $n Q\left(\frac{Q}{P}+(n-1) \frac{Q}{D}\right)-\frac{n^{2} Q^{2}}{2 P}$ and hospital's accumulated inventory is $\frac{Q^{2}}{D}(1+2+\cdots 1(n-1))$ units.

Hence, based on Uthayakumar \& Priyan [1], the pharmaceutical company's expected inventory is $E I(n, Q)=\left[\left\{n Q\left(\frac{Q}{P}+(n-1) \frac{Q}{D}\right)-\frac{n^{2} Q^{2}}{2 P}\right\}-\left\{\frac{Q^{2}}{D}(1+2+\cdots+(n-1))\right\}\right] . \quad$ Then, the company's expected holding cost per unit time $E H C(Q, P, n)=\frac{Q}{2} h_{p}\left[n\left(1-\frac{2 D}{P}\right)\right]$.

Let $g_{0}$ denote fuel consumption of the unloaded (empty) vehicle and $g_{1}$ denote unit fuel consumption factor if the supplier's transportation vehicle is loaded with goods. This research considers $g_{0}+g_{1} Q$ captures amount of fuel consumption for a one-way delivery from supplier to buyer. We assume that backhaul is not used and return vehicles are empty, and that $g_{0}$ is amount of the fuel consumption for the return trip. For one inventory replenishment, the total amount of fuel consumption $2 g_{0}+g_{1} Q$. Hence, supplier's average transportation cost per unit of time is $\frac{c D}{Q}\left(2 g_{0}+g_{1} Q\right)$

In this model, we measure the total amount of carbon emissions from transportation and storage. Thus, the associated amount of carbon emissions is designed by

$$
\begin{aligned}
& \Pi(Q, P, n)=\Pi_{1}\left(Q, P, n, t_{s}, t_{T}\right)+\Pi_{2}(Q, P, n) \\
= & n \theta_{1}\left(2 g_{0}+g_{1} Q\right)+\left(w_{0}+w_{1} \operatorname{EHC}(Q, P, n)\right) \theta_{2}
\end{aligned}
$$

In the above equation, $\Pi_{1}(Q, P, n)$ and $\Pi_{2}(Q, P, n)$ are the amounts of carbon emissions from transportation and storage, respectively. Then $\theta_{1}$ and $\theta_{2}$ are carbon emission factors for fuel and electricity. In $\Pi_{2}(Q, P, n), w_{0}$ and $w_{1}$ are fixed and unit variable electricity consumption for storage. Accordingly, the company's carbon emissions cost per unit time is derived by

$$
\Pi(Q, P, n)=\frac{D c_{0} \theta_{1}}{Q}\left(2 g_{0}+g_{1} Q\right)+\left(w_{0}+w_{1} \frac{Q}{2}\left[n\left(1-\frac{D}{P}\right)-1+\frac{2 D}{P}\right]\right) c_{0} \theta_{2}
$$

Thus, the total cost function per unit time for the pharmaceutical company comprising of setup cost, holding cost, transportation cost and carbon emission cost, is expressed by

$$
\begin{gathered}
\operatorname{ETC}_{P C}(Q, P, n)=\frac{S D}{n Q}+\frac{Q}{2} h_{p}\left[n\left(1-\frac{D}{P}\right)-1+\frac{2 D}{P}\right] \\
+\frac{D}{Q}\left(c+c_{0} \theta_{1}\right)\left(2 g_{0}+g_{1} Q\right)+\left(w_{0}+w_{1} \frac{Q}{2}\left[n\left(1-\frac{D}{P}\right)-1+\frac{2 D}{P}\right]\right) c_{0} \theta_{2}
\end{gathered}
$$

Then the joint expected total cost per unit time for the proposed pharmaceutical company and hospital integrated supply chain system is

$$
\operatorname{JETC}\left(Q, P, n, t_{s}, t_{T}\right)=\operatorname{ETC}_{P C}(Q, P, n)+\operatorname{HETC}_{b}\left(Q, p, n, t_{T}\right)
$$




$$
\begin{gathered}
=\frac{D}{Q}\left(A+\frac{s}{n}\right)+h_{b}\left(\frac{Q}{2}+\frac{1}{n} k_{1} \delta \sqrt{\frac{Q}{P}+t_{s}}+\frac{n-1}{n} k_{2} \delta \sqrt{t_{T}}\right) \\
+\frac{\varepsilon}{D}\left(1-\frac{Q}{2 D \omega}\right)+\frac{D}{n Q}\left[R\left(t_{s}\right)+(n-1) R\left(t_{T}\right)\right]+\frac{D}{Q}\left(c+c_{0} \theta_{1}\right)\left(2 g_{0}+g_{1} Q\right) \\
+w_{0} c_{0} \theta_{0}+\frac{Q}{2}\left[n\left(1-\frac{D}{P}\right)-1+\frac{2 D}{P}\right]\left(h_{p}+c_{0} \theta_{2} w_{1}\right)
\end{gathered}
$$

Now our problem involves finding the optimal $\left(Q, P, n, t_{s}, t_{T}\right)$ in a production cycle that minimize the joint expected total cost, $\operatorname{JETC}\left(Q, P, n, t_{s^{\prime}} t_{T}\right)$ while satisfying SLC. That is, the primal problems of the proposed model is given as

$$
\begin{array}{r}
\operatorname{Min} J E T C\left(Q, P, n, t_{s}, t_{T}\right)=\frac{D}{Q}\left(A+\frac{s}{n}\right)+h_{b}\left(\frac{Q}{2}+\frac{1}{n} k_{1} \sigma \sqrt{\frac{Q}{P}+t_{s}}+\frac{n-1}{n} k_{2} \sigma \sqrt{t_{T}}\right) \\
+\frac{\varepsilon}{D}\left(1-\frac{Q}{2 D}\right)+\frac{D}{n Q}\left[R\left(t_{s}\right)+(n-1) R\left(t_{T}\right)\right]+\frac{D}{Q}\left(c+c_{0} \theta_{1}\right)\left(2 g_{0}+g_{1} Q\right) \\
+w_{0} c_{0} \theta_{2}+\frac{Q}{2}\left[n\left(1-\frac{D}{P}\right)-1+\frac{2 D}{P}\right]\left(h_{p}+c_{0} \theta_{2} w_{1}\right)
\end{array}
$$

Subject to $\sigma\left(\sqrt{t_{s}+\frac{Q}{P}} \psi\left(k_{1}\right)+\sqrt{t_{T}} \psi\left(k_{2}\right)\right) \leq \alpha Q$

\section{Solution Procedure}

The problems formulated in the previous section appear as constrained non-linear programming problems. To solve these problems, we temporarily ignore the SLC and relax the integer requirement on $\mathrm{n}$, then we have to minimize joint expected total cost, $\operatorname{JETC}\left(Q, P, n, t_{\mathrm{s}^{\prime}} t_{T}\right)$

Proposition 1: For fixed $P, n, t_{s} \in\left[t_{s}^{i}, t_{s}^{i-1}\right]$ and $t_{T} \in\left[\begin{array}{ll}t_{s}^{i}, t_{s}^{i-1}\end{array}\right]$, $\operatorname{JETC}\left(Q, P, n, t_{s} t_{T}\right)$ is convex in $Q$.

Proof: Taking the first and second partial derivatives of $\operatorname{JETC}\left(Q, P, n, t_{\mathrm{S}} t_{T}\right)$ with respect to $Q$ we have

$$
\begin{gathered}
\frac{\partial \operatorname{JETC}\left(Q, P, n, t_{s}, t_{T}\right)}{\partial Q}=-\frac{D}{Q^{2}}\left(A+\frac{S}{n}\right)+\frac{h_{b}}{2}\left(1+\frac{1}{n} k_{1} \sigma \frac{1}{\sqrt{\frac{Q}{P}+t_{s}}}\right) \\
-\frac{\varepsilon}{2 D^{2} \omega}-\frac{D}{n Q^{2}}\left[R\left(t_{s}\right)+(n-1) R\left(t_{T}\right)\right]-\frac{2 g_{0} D}{Q^{2}}\left(c+c_{0} \theta_{1}\right) \\
\frac{1}{2}\left[n\left(1-\frac{D}{P}\right)-1+\frac{2 D}{P}\right]\left(h_{p}+c_{0} \theta_{2} w_{1}\right)
\end{gathered}
$$

and

$$
\begin{aligned}
& \frac{\partial^{2} \operatorname{JETC}(Q, L, n)}{\partial Q^{2}}=-\frac{2 D}{Q^{2}}\left(A+\frac{S}{n}\right)+\frac{h_{b}}{2} k_{1} \sigma\left(\frac{Q}{P}+t_{s}\right)^{-\frac{3}{2}} \\
& +\frac{2 D}{n Q^{3}}\left[R\left(t_{s}\right)+(n-1) R\left(t_{T}\right)\right]+\frac{4 g_{0} D}{Q^{3}}\left(c+c_{0} \theta_{1}\right)>0
\end{aligned}
$$

Therefore, for fixed $P, n, t_{s} \in\left[t_{s}^{i}, t_{s}^{i-1}\right]$ and $t_{T} \in\left[t_{s}^{j}, t_{s}^{j-1}\right]$, is convex in $Q$.

This completes the proof of Proposition 1.

Result 1: For the given values of $P, n, t_{s} \in\left[t_{s}^{i}, t_{s}^{i-1}\right]$ and $t_{T} \in\left[t_{s}^{j}, t_{s}^{j-1}\right]$, by setting Eq. (2) equal to zero, we obtain the optimal $Q$ and $Q_{H}$ which minimize the $\operatorname{JETC}\left(Q, P, n, t_{S}, t_{T}\right)$ :

$$
Q_{H}=\left\{\frac{2 D\left[A n+s+\left[R\left(t_{s}\right)+(n-1) R\left(t_{T}\right)\right]+2 n g_{0}\left(c+c_{0} \theta_{1}\right)\right]}{\left[h_{b}\left(1+\frac{1}{n} k_{1} \sigma\left(\frac{Q}{P}+t_{s}\right)^{-\frac{1}{2}}\right]-\frac{\varepsilon}{D^{2} \omega}+\left[n\left(1-\frac{D}{P}\right)-1+\frac{2 D}{P}\right]\left(h_{p}+c_{0} \theta_{2} w_{1}\right)\right]}\right\}^{\frac{1}{2}}
$$

Proposition 2: For fixed $Q, n, t_{s} \in\left[t_{s^{\prime}}^{i} t_{s}^{i-1}\right]$ and $t_{T} \in\left[t_{s^{j}}, t_{s}^{j-1}\right]$, $\operatorname{JETC}\left(Q, P, n, t_{s}, t_{T}\right)$ is convex in $P$.

Proof: Taking the first and second partial derivatives of $\operatorname{JETC}\left(Q, P, n, t_{\mathrm{S}} t_{T}\right)$ with respect to $P$, we have

$$
\frac{\partial \operatorname{JETC}\left(Q, P, n, t_{s}, t_{T}\right)}{\partial P}=-\frac{h_{b}}{2 n} k_{1} \sigma\left(\frac{Q}{P}+t_{s}\right)^{-\frac{1}{2}} \frac{Q}{P^{2}}+\frac{n Q D}{2 P^{2}}-\frac{Q D}{P^{2}}\left(h_{p}+c_{0} \theta_{2} w_{1}\right)
$$

and

$\frac{\partial \operatorname{JETC}\left(Q, P, n, t_{s}, t_{T}\right)}{\partial P}=-\frac{h_{b}}{2 n} k_{1} \sigma\left[\frac{2 Q}{P^{3}}\left(\frac{Q}{P}+t_{s}\right)^{-\frac{1}{2}}\left\{\frac{Q}{4 P}\left(\frac{Q}{P}+t_{s}\right)^{-2}-1\right\}\right]+\frac{2 Q D}{P^{3}}\left(h_{p}+c_{0} \theta_{2} w_{1}\right)>0$

Therefore, for fixed $P, n, t_{s} \in\left[\begin{array}{ll}t_{s}^{i}, & \left.t_{s}^{i-1}\right]\end{array}\right]$ and $t_{T} \in\left[\begin{array}{ll}t_{s}^{j}, & t_{s}^{j-1}\end{array}\right]$, $\operatorname{JETC}\left(Q, P, n, t_{s} t_{T}\right)$ is convex in $P$. This completes the proof of Proposition 2.

Result 1: For the given values of $Q, n, t_{s} \in\left[t_{s}^{i}, t_{s}^{i-1}\right]$ and $t_{s} \in\left[t_{s}^{i}, t_{s}^{i-}\right.$ ${ }^{1}$ ], by setting Eq. (4) equal to zero, we obtain the optimal $P$ which minimize the $\operatorname{JETC}\left(Q, P, n, t_{s}, t_{T}\right)$ :

Proposition 3: For fixed $Q, P, n J E T C\left(Q, P, n, t_{s} t_{T}\right)$ is concave in $t_{s}$ $\epsilon\left[t_{s}^{i}, t_{s}^{i-1}\right]$ and $t_{T} \in\left[t_{s}^{j}, t_{s}^{j-1}\right], i=1,2, \ldots, m$ and $j=1,2, \ldots, m-1$

Proof: Taking the second partial derivatives of $\operatorname{JETC}\left(Q, P, n, t_{s}, t_{T}\right)$ with respect to $t_{s}$ and $t_{T}$ respectively, we obtain the following Hessian matrix

$$
H=\left(\begin{array}{cc}
\frac{\sigma k_{1} h_{b}}{4 n}\left(\frac{Q}{P}+t_{s}\right)^{\frac{3}{2}} & 0 \\
0 & \frac{1}{4 n}(n-1) \sigma k_{2} t_{T}^{\frac{3}{2}}
\end{array}\right)
$$

It is clear that the Hessian matrix $\mathrm{H}$ is negative definite. Hence, $J E T C\left(Q, P, n, t_{s} t_{T}\right)$ is a concave function with respect to $t_{s}$ and $t_{T}$ for fixed values of $Q, P, n$.

This completes the proof of Proposition 3.

Result 2: According to Proposition 3, for fixed $Q, P, n$, the minimum values of $\operatorname{JETC}\left(Q, P, n, t_{s}, t_{T}\right)$ and occur at the end points of the interval is $t_{s} \in\left[t_{s}^{i}, t_{s}^{i-1}\right] X t_{T} \in\left[t_{s}^{j}, t_{s}^{j-1}\right]$

Proposition 4: For fixed $Q, P, n, t_{s} \in\left[t_{s}^{i}, t_{s}^{i-1}\right]$ and $t_{T} \in\left[t_{s}^{j}, t_{s}^{j-1}\right]$, $\operatorname{JETC}\left(Q, P, n, t_{s^{\prime}} t_{T}\right)$ is convex in

Proof: Taking the first and second partial derivatives of $\operatorname{JETC}\left(Q, P, n, t_{s}, t_{T}\right)$ with respect to we have

$$
\begin{aligned}
& \frac{\partial \operatorname{JETC}(Q, L, n)}{\partial n}=\frac{D S}{Q n^{2}}-\frac{h_{b}}{n^{2}} k_{1} \sigma \sqrt{\frac{Q}{P}+t_{s}}+\frac{1}{n^{2}} k_{2} \sigma \sqrt{t_{T}}-\frac{D}{n^{2} Q}\left[R\left(t_{s}\right)-R\left(t_{T}\right)\right]+\frac{Q}{2} h_{p}\left(1-\frac{D}{P}\right) \\
& \quad \text { and } \\
& \frac{\partial^{2} \operatorname{JETC}(Q, L, n)}{\partial n}=\frac{2 D S}{Q n^{3}}-\frac{2 h_{b}}{n^{3}} k_{1} \sigma \sqrt{\frac{Q}{P}+t_{s}}-2 \frac{1}{n^{3}} k_{2} \sigma \sqrt{t_{T}}-\frac{D}{n^{3} Q}\left[R\left(t_{s}\right)-R\left(t_{T}\right)\right]>0
\end{aligned}
$$

Therefore, for fixed $Q$ and $L \in\left[L_{i}, L_{i-1}\right]$, JETC $(Q, n, L)$ is convex in $n$. 
This completes the proof of Proposition 4.

Remark 1: The search for the optimal number of deliveries, is reduced to find a local minimum.

According to the Result 1, for fixed of $P, n, t_{s} \in\left[t_{s}^{i}, t_{s}^{i-1}\right]$ and $t_{T} \in\left[t_{s}^{j}\right.$, $\left.t_{s}^{j-1}\right]$, when the SLC is ignored, Eq. (3) gives optimal value of $Q$ such that the joint expected total cost is minimum. Now, the SLC is taken into consideration and if for $Q=Q_{H}$ then $Q_{H}$ is the local minimum of $\operatorname{JETC}\left(Q, P, n, t_{s}, t_{T}\right)$ and SLC is inactive. Otherwise, optimal value of $Q$ should be at least equal to $Q_{S L C}=(\sigma / \alpha)\left(\sqrt{t_{s}+\frac{Q}{P}} \psi\left(k_{1}\right)+\sqrt{t_{T}} \psi\left(k_{2}\right)\right)$ which is greater than $Q_{H}$ so that specified level of service can be achieved at minimum joint expected total cost and $Q_{S L C}$ is the local minimum of $\operatorname{JETC}\left(Q, P, n, t_{s} t_{T}\right)$. So, for fixed of $P, n, t_{s} \in\left[t_{s}^{i}, t_{s}^{i-}\right.$ $\left.{ }^{1}\right]$ and $t_{T} \in\left[t_{s}^{j}, t_{s}^{j-1}\right]$ the optimal $Q$ is given by $\max \left\{Q_{H} Q_{S L C}\right\}$. Based on the convexity and concavity behavior of objective function with respect to the decision variables the following algorithm is designed to find the optimal values of the decision variables.

\section{Algorithm}

i. Step 1. Set $n=1$.

ii. Step 2. For each $t_{s}^{i}$ and $t_{s}^{j}$ perform (2-1) to (2-5), $i=0,1, \ldots, m$ and $j=0,1, \ldots, m-1$.

- $\quad$ Step 2.1. Compute $P_{i j}=\sqrt{\frac{a_{1}}{a_{2}}}$ and $Q_{H}$ using Eq. (3).

- $\quad$ Step 2.2. Compute $p_{i j}^{\prime}$ and $Q_{H}^{\prime}$ using Result 1 and Eq. (3) respectively. Repeat this step until convergence. Then set the optimal values $p_{i j}^{\circ}$ and $Q^{\circ}{ }_{H}$

- $\quad$ Step 2.3 Set $Q=\max \left\{Q^{\circ}{ }_{H} Q_{S L C}\right\}$. If $Q_{H}^{\circ}$ is maximum, then go to step 2.5, otherwise go to step 2.4.

- $\quad$ Step 2.4. Compute $p^{\circ}{ }_{i j}$ using $Q_{S L C}$ then go to step 2.5.

- $\quad$ Step 2.5. Compute the corresponding $\operatorname{JETC}\left(Q_{i j^{\prime}} P_{i j^{\prime}} n, t_{s^{\prime}} t_{T}\right)$ by putting $Q=Q^{\circ}{ }_{H}$ and $p=p^{\circ}{ }_{i j}$ in Eq. (1).

iii. Step 3. Let $\operatorname{JETC}\left(Q^{*}, P^{*}, n, t_{s}^{*}, t_{T}^{*}\right)=\min _{(i=0,1, \ldots, m-1),(j=0,1, \ldots, m-1)}$ $\operatorname{JETC}\left(Q_{i p^{\prime}} P_{i p^{\prime}} n, t_{s^{\prime}} t_{T}\right)$. Then the optimal solution for fixed values of $n$ is $\left(Q^{*}, P^{*}, n, t_{s}^{*}, t_{T}^{*}\right)$.

iv. Step 4: Set $n=n+1$

v. Step 5: Repeat Steps 3 and 3 to get $\operatorname{MJETC}\left(Q^{*}, P^{*}, n, t_{s}^{*}, t_{T}^{*}\right)$.

vi. Step 6: If $\operatorname{JETC}\left(Q^{*}, P^{*}, n, t_{s}^{*}, t_{T}{ }^{*}\right) \leq \operatorname{JETC}\left(Q^{*}, P^{*}, n-1, t_{s}^{*}, t_{T}{ }^{*}\right)$ then go to Step 4 , otherwise go to Step 7

vii. Step 7: Set $\left(Q^{*}, P^{*}, n, t_{s}^{*}, t_{T}^{*}\right)=\left(Q^{*}, P^{*}, n-1, t_{s}^{*}, t_{T}^{*}\right)$. Then $\left(Q^{*}, P^{*}, n, t_{s}^{*}, t_{T}^{*}\right)$ is optimal solution to achieve target CSL which minimize joint expected total cost.

\section{Conclusion}

In this paper, we designed a new algorithm to achieve the target customer service level while minimizing system cost of the proposed healthcare supply chain system. We provided an optimal inventory policy through mathematical model to modify the operators in order to reduce carbon emissions. This research explores the impact of both lead time and carbon emissions on the inventory decisions of a pharmaceutical company and hospital supply chain system.

\section{References}

1. Uthayakumar R, Priyan S (2013) Pharmaceutical supply chain and inventory management strategies: optimization for a pharmaceutical company and a hospital. Operations Research for Health Care 2(3): 5264.

2. Priyan S, Uthayakumar R (2014) Optimal inventory management strategies for pharmaceutical company and hospital supply chain in a fuzzy-stochastic environment. Operations Research for Health Care 3(4): 177-190.

3. Susarla N, Karimi IA (2018) Integrated production planning and inventory management in a multinational pharmaceutical supply chain. Computer Aided Chemical Engineering 41: 551-567.

4. Savadkoohi E, Mousazadeh M, Ali Torabi S (2018) A possibilistic location-inventory model for multi-period perishable pharmaceutical supply chain network design. Chemical Engineering Research and Design 138: 490-505.

5. Kim D (2005) An integrated supply chain management system: a case study in healthcare sector. In: Lecture Notes on Computational Science 3590: 218-227.

6. Bert Meijbooma, Børge Obel (2007) Tactical coordination in a multi-location and multi-stage operations structure: a model and a pharmaceutical company case. Omega 35(3): 258-273.

7. Amir H Masoumi, Min Yu, Anna Nagurney (2012) A supply chain generalized network oligopoly model for pharmaceuticals under brand differentiation and perishability. Transportation Research E 48(4): 762-780.

8. Liao CJ, Shyu CH (1991) An analytical determination of lead time with normal demand. International Journal of Operations Production Management 11: 72-78.

9. Pan JCH, Yang JS (2002) A study of an integrated inventory with controllable lead time. International Journal of Production Research 40: 1263-1273.

10. Liang Yuh Ouyang, Kun Shan Wu, Chia Huei Ho (2004) Integrated vendor buyer cooperative models with stochastic demand in controllable lead time. International Journal of Production Economics 92(3): 255-266.

11. Mohammad A Hoque, Suresh K Goyal (2006) A heuristic procedure for an integrated inventory system under controllable lead-time with equal or unequal sized batch shipments between a vendor and a buyer. International Journal of Production Economics 102(2): 217-225.

12. Hoque MA (2007) An alternative model for integrated vendor buyer inventory under controllable lead time and its heuristic solution. International Journal of Systems Science 38: 501-509.

13. Glock CH (2012) Lead time reduction strategies in a single-vendorsingle-buyer integrated inventory model with lot size-dependent lead times and stochastic demand. Int J Production Economics 136: 37-44.

14. Moua Q, Cheng Y, Liao H (2017) A note on lead time reduction strategies in a single-vendor-single-buyer integrated inventory model with lot size-dependent lead times and stochastic demand. International Journal of Production Economics 193: 827-831.

15. Jaber MY, Glock CH, El Saadany AMA (2013) Supply chain coordination with emissions reduction incentives. International Journal of Production Research 51: 6982.

16. Hammami R, Nouira I, Frein Y (2015) Carbon emissions in a multiechelon production-inventory model with lead time constraints. International Journal of Production Economics 164: 292-307. 
17. Jauhari WA, Pamuji AS, Rosyidi CN (2014) Cooperative inventory model for vendor-buyer system with unequal sized shipment, defective items and carbon emission cost. International Journal of Logistics Systems and Management 19: 163-186.

18. Sunil Tiwari, Yosef Daryanto, Hui Ming Wee (2018) Sustainable inventory management with deteriorating and imperfect quality items considering carbon emission. Journal of Cleaner Production 192: 281292.

19. Qingguo Bai, Yeming Gong, Mingzhou Jin, Xianhao Xu (2019) Effects of carbon emission reduction on supply chain coordination with vendormanaged deteriorating product inventory. International Journal of Production Economics 208: 83-99.

\section{Your next submission with Juniper Publishers will reach you the below assets}

- Quality Editorial service

- Swift Peer Review

- Reprints availability

- E-prints Service

- Manuscript Podcast for convenient understanding

- Global attainment for your research

- Manuscript accessibility in different formats

( Pdf, E-pub, Full Text, Audio)

- Unceasing customer service

Track the below URL for one-step submission https://juniperpublishers.com/online-submission.php 